\title{
IO Aqr: Twins just at turn off?^
}

\author{
W. Dimitrov ${ }^{1}$, D. Kolev ${ }^{2}$, and A. Schwarzenberg-Czerny ${ }^{1,3}$ \\ 1 Astronomical Observatory of Adam Mickiewicz University, ul. Słoneczna 36, 60-286 Poznań, Poland \\ 2 National Astronomical Observatory Rozhen, PO Box 136, 4700 Smolyan, Bulgaria \\ e-mail: dzkolev@abv.bg \\ ${ }^{3}$ Copernicus Astronomical Center, ul. Bartycka 18, 00-716 Warszawa, Poland \\ e-mail: alex@camk.edu.pl
}

Received 22 April 2003 / Accepted 8 December 2003

\begin{abstract}
We present photometric and spectroscopic analysis of the eclipsing binary IO Aqr with orbital period 2.37. The system is detached, with similar almost spherical components. The star is classified as G0 spectral type. In the analysis we have used spectra obtained at a 2-m telescope with a Coudé spectrograph for two regions $\mathrm{NaD}$ and $\mathrm{H}_{\alpha}$, as well as ASAS $I$ band photometry and $R, V$ photometry obtained at a $0.4 \mathrm{~m}$ telescope. The model of the star was obtained using a code based on the Wilson-Devinney method. The radii of the stars for our best fit are $R_{1}=2.09 \pm 0.13 R_{\odot}$ and $R_{2}=2.54 \pm 0.06 R_{\odot}$ while the masses of the components are $M_{1}=1.53 \pm 0.02 M_{\odot}$ and $M_{2}=1.64 \pm 0.02 M_{\odot}$. Both components are slightly evolved main sequence stars.
\end{abstract}

Key words. eclipsing binaries - binaries: spectroscopic - stars: individual: IO Aqr

\section{Introduction}

The detached eclipsing binary IO Aqr (BD+004569) is listed among the Hipparcos stars with the largest photometric amplitude (HIC 102041). Its extensive photometry was listed by ASAS (All Sky Automated Survey) (Pojmański 1997) under code ASAS 204045+0056.4. Its magnitude at maximum is $V_{\max }=8.87$. Casual inspection of the light curve reveals two eclipses of nearly identical depth and some ellipsoidal distortion at maximum. These characteristics suggested that IO Aqr might be a detached binary of two nearly identical components.

The photometric observations obtained by Hipparcos yielded its orbital period of 2.36816 (Perryman et al. 1997). The color index as obtained in these observations corresponds to $B-V=0.512 \pm 0.025$. The color index in the Vilnius system (Cernis 1986), after reduction to the Johnson system, yields $B-V=0.53$. The value given by Tycho 2 is $B-V=0.45 \pm 0.03$. The star is listed in the Henry Draper catalogue as G0 spectral type. The corresponding color indices for main sequence and giant G0 stars are $B-V=0.58$ and 0.65 , respectively. The Hipparchos parallax of IO Aqr amounting to $5.4 \pm 1.3$ mas and the $V$ magnitude suggest that the components have bigger radii than a G0 main sequence star. The proper motion of IO Aqr is $22 \pm 2$ milliarcseconds per year.

Send offprint requests to: $\mathrm{W}$. Dimitrov,

e-mail: dimitrov@amu.edu.pl

* Based on spectroscopic observations collected at National Astronomical Observatory Rozhen, Bulgaria and David Dunlop Observatory, Canada.
The Hipparcos parallax and the rather uncomplicated light curve of IO Aqr make this star a good candidate for the exact determination of the stellar parameters and for the calibration of the photometric parallax (Kruszewski \& Semeniuk 1999). Although the eclipses of IO Aqr are partial, the radiusinclination indeterminacy might be avoided if ellipsoidal distortion imposes sufficiently strict limits on the radii. Since a search of the literature with Simbad revealed no radial velocity curve for this star, we resorted to own observations. Our data and the corresponding ephemeris are presented in Sects. 2 and 3, respectively. In Sect. 4 we fit observations of the binary with the Wilson-Devinney model. Section 5 is devoted to discussion of the age and distance of the system.

\section{Data}

\subsection{Spectroscopy}

We obtained spectra of IO Aqr at the Bulgarian National Astronomical Observatory Rozhen, with the $2.0 \mathrm{~m}$ RCC telescope with Coudé spectrograph during 7 nights in August 2001. The detector was an LN-cooled SITE $1 \mathrm{~K} \times 1 \mathrm{~K}, 24 \mu \mathrm{m}$ CCDmatrix. We observed the two spectral regions of $\mathrm{NaD}$ and $\mathrm{H}_{\alpha}$ covering the wavelength ranges from $5800 \AA$ to $6000 \AA$ and from $6450 \AA$ to $6650 \AA$ with a dispersion of $0.2 \AA$ per pixel. The exposure times were $1200 \mathrm{~s}$ and the typical signal-to-noise ratio is 80 . The wavelength calibration was carried out using a ThAr hollow cathode lamp. The typical standard deviation is $\sigma=0.02 \AA$, providing a radial velocity accuracy of about 
Table 1. Radial velocity measurements for $\mathrm{NaD}$ region. Outlying points marked by a star $(\star)$ were not used in the further analysis.

\begin{tabular}{cccccc}
\hline \hline $\begin{array}{c}\text { HJD } \\
2452100+\end{array}$ & $\begin{array}{c}R V 1 \\
\mathrm{~km} \mathrm{~s}^{-1}\end{array}$ & $\begin{array}{c}(\mathrm{O}-\mathrm{C})_{1} \\
\mathrm{~km} \mathrm{~s}^{-1}\end{array}$ & $\begin{array}{c}R V 2 \\
\mathrm{~km} \mathrm{~s}^{-1}\end{array}$ & $\begin{array}{c}(\mathrm{O}-\mathrm{C})_{2} \\
\mathrm{~km} \mathrm{~s}^{-1}\end{array}$ & phase \\
\hline 26.2722 & -111.5 & -1.0 & +100.1 & +0.6 & 0.318 \\
26.2865 & -108.1 & +0.5 & +98.0 & +0.4 & 0.324 \\
26.4205 & -82.7 & +0.7 & $+84.0^{\star}$ & +10.0 & 0.381 \\
26.4356 & -78.3 & +1.2 & $+79.0^{\star}$ & +8.7 & 0.387 \\
27.2979 & +120.2 & +2.9 & -118.4 & -4.4 & 0.751 \\
27.3126 & +120.7 & +3.5 & -117.7 & -3.8 & 0.758 \\
27.4172 & +111.6 & +0.6 & -108.0 & +0.1 & 0.802 \\
27.4315 & +111.4 & +1.9 & -107.8 & -1.1 & 0.808 \\
27.5419 & +94.3 & +2.0 & -86.8 & +3.8 & 0.854 \\
27.5561 & +90.0 & +0.5 & $-78.7^{\star}$ & +9.3 & 0.860 \\
28.3861 & -120.7 & -2.8 & +103.9 & -2.6 & 0.211 \\
28.4042 & -121.9 & -2.6 & +106.2 & -1.5 & 0.218 \\
28.4672 & -121.0 & +0.5 & +108.3 & -1.5 & 0.245 \\
28.4826 & -123.6 & -2.1 & +108.9 & -0.9 & 0.252 \\
28.4982 & -118.6 & +2.7 & +107.9 & -1.7 & 0.258 \\
29.3796 & +86.4 & +0.8 & -80.0 & +4.2 & 0.630 \\
29.3938 & +87.2 & -1.3 & -83.8 & +3.2 & 0.636 \\
29.5155 & +105.9 & -2.6 & -109.5 & -3.9 & 0.688 \\
29.5302 & +107.1 & -2.9 & -110.1 & -3.0 & 0.694 \\
30.4702 & -62.5 & +4.3 & $+70.9^{\star}$ & +12.4 & 0.091 \\
30.4851 & -71.3 & -0.1 & $+74.1^{\star}$ & +11.5 & 0.097 \\
30.5919 & -92.8 & +2.8 & +86.5 & +1.0 & 0.142 \\
30.6066 & -94.6 & +3.6 & +86.2 & -1.9 & 0.149 \\
\hline & & & & &
\end{tabular}

$1 \mathrm{~km} \mathrm{~s}^{-1}$. Unfortunately, we were not able to obtain $\mathrm{H}_{\alpha}$ frames in all of the phases, but the $\mathrm{H}_{\alpha}$ data confirmed to a large extent the result shown by the spectra near the $\mathrm{NaD}$ lines. All spectra were processed with the standard IRAF procedures by one of the authors (W.D.). For the $\mathrm{NaD}$ data we have used a crosscorrelation function (IRAF task FXCOR) to determine the line shifts for the two components. We have used one of our spectra - very close to phase zero - as a template. The heliocentric velocity of our template was computed with the IRAF task RVIDLINES. The stars are similar, so we see clearly lines from both components in the spectra. The mean square error of the results listed in Table 1 (without outliers) for the first component is $2.2 \mathrm{~km} \mathrm{~s}^{-1}$ and for the second component $2.5 \mathrm{~km} \mathrm{~s}^{-1}$.

The NaD spectrograms reveal (Fig. 1) lines from both components as well as a third line, probably a result of interstellar absorption. These $\mathrm{NaD}_{1}$ and $\mathrm{NaD}_{2}$ lines make the radial velocity measurements more complicated because we also have a third peak in the CCF function. The mean radial velocity of the third line is $-17 \mathrm{~km} \mathrm{~s}^{-1}$. The $F W H M$ of this line approximates the instrumental one $(0.4 \AA)$.

The reduction of the $\mathrm{H}_{\alpha}$ observations was carried out with the IRAF RVIDLINES task (Fig. 2). In order to check our results we also used a few spectral frames obtained by Piotr Ligeza at DDO (Canada) near the Calcium triplet (5175 A) well suitable for radial velocity analysis.

\subsection{Photometry}

We have a light curve of 89 points collected during a nearly three years period of observations by Hipparcos. Unfortunately,

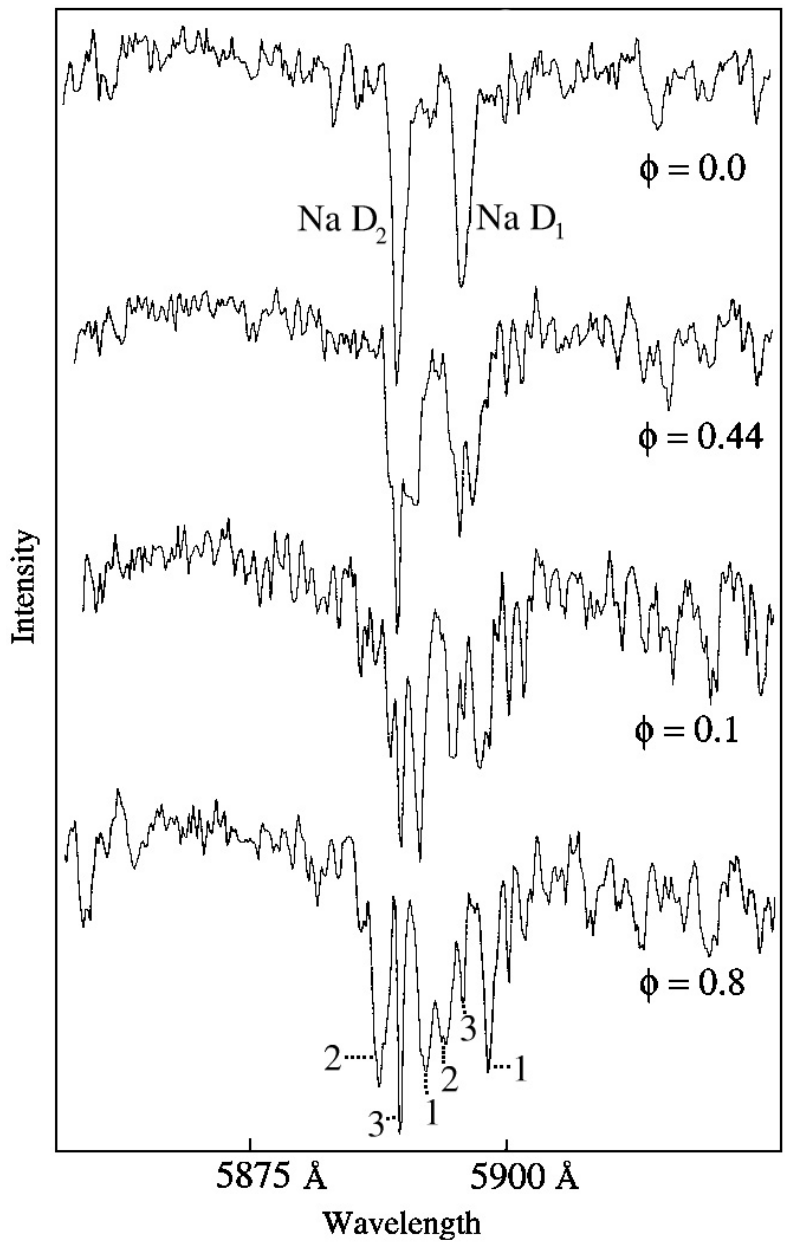

Fig. 1. Examples of spectra in $\mathrm{NaD}$ region for different phases. We can see lines from both components $(1,2)$ and a third line (3) probably connected with interstellar absorption.

the phase coverage is not good enough especially for the primary minimum.

The light curve from ASAS is of good quality, covers all phases and contains 992 I-band points collected in over a year. Its amplitude is 0.37 and the corresponding orbital period is 2.368131 days. The individual ASAS errors are large, as expected for a small telescope with imperfect PSF, and depend on magnitude and location. However, the excellent distribution of our normal points demonstrates the good calibration and stability of ASAS photometry. We back-calculate the individual error from our $\mathrm{O}-\mathrm{C}$ of normal points to be about $0.015 \mathrm{mag}$. To update the ephemeris, we undertook photometric observations at the Borowiec Astronomical Observatory (Poland), using the $0.4 \mathrm{~m}$ Newton telescope with a KAF 400 CCD camera $(750 \times 510$ pixels) and $R$ and $V$ filters (Table 2). We used the Starlink package from CCLR for reduction and aperture photometry . The differential photometry was obtained using as comparison stars GSC 0511-0962 $\left(V=10^{\mathrm{m}} \cdot 7\right)$ and GSC 0511-2041 ( $V=11$ m 0 ). The typical errors of Borowiec IO Aqr photometry are about $0 \mathrm{~m} 01$. The phase coverage of these observations was incomplete but nevertheless they enabled us to upgrade the ephemeris (Sect. 3). 


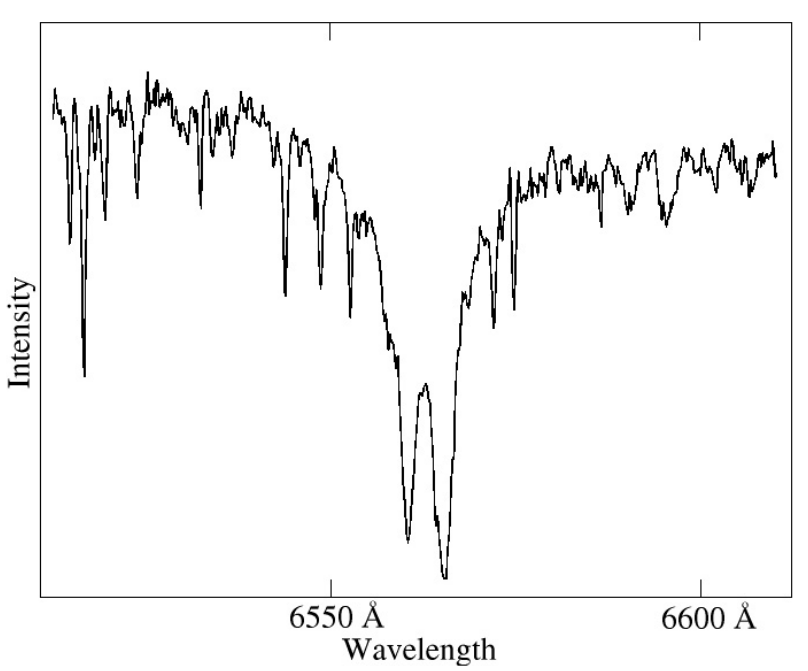

Fig. 2. Part of the $\mathrm{H}_{\alpha}$ spectrogram for phase 0.23. Radial velocities measured (only from the $\mathrm{H}_{\alpha}$ line) for the two components are -108.3 and $105.5 \mathrm{~km} \mathrm{~s}^{-1}$.

Table 2. Log of photometric observations of IO Aqr.

\begin{tabular}{cccccc}
\hline \hline Date & $\begin{array}{c}T \text { start } \\
\text { UT }\end{array}$ & $\begin{array}{c}T \text { end } \\
\text { UT }\end{array}$ & Filter & $\begin{array}{c}\text { Exp. time } \\
\text { s }\end{array}$ & No \\
& $21: 40$ & $1: 30$ & $R$ & 45 & 202 \\
\hline 2002 July 21 & $R: 30$ & $1: 45$ & $R$ & 45 & 276 \\
2002 July 28 & $20: 30$ & & \\
2002 July 31 & $21: 00$ & $1: 30$ & $R$ & 40 & 261 \\
2002 Aug. 3 & $20: 18$ & $1: 20$ & $R$ & 45 & 270 \\
2002 Sep. 12 & $19: 30$ & $23: 00$ & $V$ & 100 & 97 \\
\hline
\end{tabular}

\section{The ephemeris}

Hipparcos yields an orbital period of 2.36816 days based on data spanning 2.65 years. The ASAS observations are of higher quality but span only 1.26 year. They quote a period of 2.368131 with no explicit value for an error. However, according to the photometry quality, the phases must be established with an accuracy of about $0.01 \mathrm{P}$. For a one year time span this corresponds to an period error of $\pm 6 \times 10^{-5}$. The two photometric periods agree at this accuracy level. Our photometry was obtained more than three years after ASAS. The corresponding phase uncertainty does not exceed 0.05 , hence our cycle count is secure. We assume a circular orbit. We use photometric observations only from ASAS and Borowiec to improve the ephemeris of IO Aqr. We obtain the following value $P=2.368091 \pm 8 \times 10^{-6}$ and the ephemeris:

$\min I=\operatorname{HJD} 2452530.4516+2.368091 \cdot E$

where the uncertainty of the primary transit is 0.0020 . The ephemeris agrees well with the times of minima from different sources of photometric and spectroscopic data (Table 3). Phase zero corresponds to the time of the deeper minimum, the eclipse of the lighter (1) by the more massive star (2).
Table 3. Times of minima of IO Aqr from photometric and spectroscopic observations.

\begin{tabular}{cccc}
\hline \hline Date & HJD & Minimum & Source \\
\hline 2 Sep. 1991 & 2448502.3278 & I & Hipparcos (phot.) \\
31 May 1999 & 2451329.8295 & I & ASAS (phot.) \\
4 Aug. 2001 & 2452125.5187 & I & Rozhen (spectr.) \\
12 Sep. 2002 & 2452530.4516 & I & Borowiec (phot.) \\
\hline
\end{tabular}

Table 4. Orbital parameters of IO Aqr.

\begin{tabular}{|c|c|c|}
\hline Parameter & Star 1 & Star 2 \\
\hline fitted: & & \\
\hline$K\left(\mathrm{~km} \mathrm{~s}^{-1}\right)$ & $119.6 \pm 0.5$ & $111.9 \pm 0.6$ \\
\hline$V_{\gamma}\left(\mathrm{km} \mathrm{s}^{-1}\right)$ & \multirow{2}{*}{\multicolumn{2}{|c|}{$-2.2 \pm 0.6$}} \\
\hline calculated: & & \\
\hline$A \sin i\left(R_{\odot}\right)$ & \multicolumn{2}{|c|}{$10.830 \pm 0.036$} \\
\hline$A_{1,2} \sin i\left(10^{6} \mathrm{~km}\right)$ & $3.894 \pm 0.015$ & $3.644 \pm 0.020$ \\
\hline $\begin{array}{l}M_{1,2} \sin ^{3} i\left(M_{\odot}\right) \\
\text { other quantities: }\end{array}$ & $1.474 \pm 0.017$ & $1.575 \pm 0.015$ \\
\hline$N_{\text {obs }}$ & 23 & 18 \\
\hline$\sigma\left(\mathrm{km} \mathrm{s}^{-1}\right)$ & 2.2 & 2.5 \\
\hline
\end{tabular}

\section{Stellar parameters}

\subsection{Input data}

Radial velocity curves, obtained from NaD (Table 1) measurements only, were fitted with sine functions (Table 4). Five outlying points were omitted in the process (see Sect. 4.2). The system velocity $V_{\gamma}$ was calculated as an average from both curves.

The light curve maxima are slightly curved, consistent with some ellipticity of the detached components (Fig. 3). The curvature of both minima and the fact that their depth reaches no more than 0.4 indicates that the eclipses are partial. Similar amplitudes of the radial velocity curves indicate similar masses of components. It is also natural to assume the same age for both members of the binary. Using the color index $B-V=$ $0.45 \pm 0.03$ given in the Tycho 2 catalogue, we can assume that the temperature $T_{1}$ of the first component is equal to $6600 \mathrm{~K}$. From trial runs of the W-D code with the temperature of the second component relaxed we saw that $T_{1}$ and $T_{2}$ should be very close to each other (see Sect. 4.2). The similarity of temperatures, masses, and ages for any conventional evolutionary scheme suggests similar radii and luminosities. We assume that for both stars the gravity darkening coefficient is 0.32 (convective envelope) and the albedo is 0.5 . According to Wade \& Rucinski 1985 for $\log (g)=3.9$ we adopted an $I$-band limb darkening coefficient of 0.4 .

Because it has the best coverage, we selected the ASAS $I$-band light curve for modeling. The $I$-band photometric observations were binned into normal points with due care for their individual weights. The range and spacing of the normal points during eclipses was decreased four-fold. A different density of the normal points was adopted as a compromise between coverage and statistics at a given phase. The adopted unit of luminosity corresponded to $I_{0}=8.27$. 

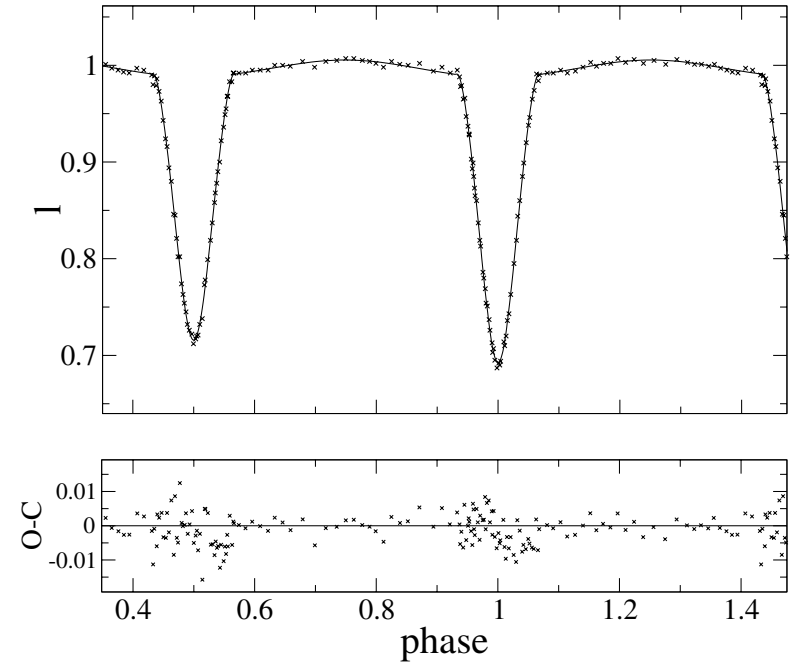

Fig. 3. Light curve of IO Aqr for solution A. There is no diference between the two solutions on this scale. We compare the synthetic light curve with the normal points for I-band ASAS photometry. For better coverage of the curve we use denser normal points during eclipses. At phase 0 component 1 is being eclipsed by component 2 .

To assess the uniqueness of the solution and the global nature of the $\chi^{2}$ minimum we performed many trial runs starting from different initial value sets of the fixed and adjustable parameters. We tested two types of solutions, based on the photometry alone and on the combined photometric and radial velocity data respectively.

\subsection{Wilson-Devinney model}

To determine the stellar parameters we employ the Wilson-Devinney (1971) code for the synthesis of the light and radial velocity curves wrapped up in the optimization package of Plewa $(1992,1988)$. Trial fits with the temperature of the secondary star $T_{2}$, relaxed, suggest that the first component is hotter by $200 \pm 300 \mathrm{~K}$.

After some experiments, we decide to use only the $\mathrm{NaD}$ spectra. In the final solution we rejected the radial velocities outlying by more than $8 \mathrm{~km} \mathrm{~s}^{-1}$ marked in Table 1 . This procedure affected the mass values by less than $2 \%$ but halved their errors, so for the final solution we use 23 points for the primary component and 18 for the secondary. Using three DDO spectrograms alone we found a mass ratio of $1.073 \pm 0.05$ that is consistent within errors with the one obtained at NAO Rozhen.

Table 5 presents our two solutions (A and B). The synthetic light curves for both of them are almost identical (Fig. 3). Although the first solution has a smaller $\chi^{2}$ the second agrees better with the evolutionary tracks (Fig. 5). The final fit (Tables 5,6) for A yields nearly identical components with mass ratio $q$ and radius ratio $R_{2} / R_{1}$ equal to 1.068 and 1.044 respectively. In the case of $\mathrm{B}$ the mass ratio is the same while $R_{2} / R_{1}$ is significantly bigger and equal to 1.215 . Neither of the components fills its Roche lobe and both of them have noticeable ellipsoidal distortion. The corresponding fill factors for A are $f_{1}=-3.52$ and $f_{2}=-3.73$, as defined by Wilson $\&$ Devinney, and for solution $\mathrm{B} f_{1}=-4.29$ and $f_{2}=-3.18$.

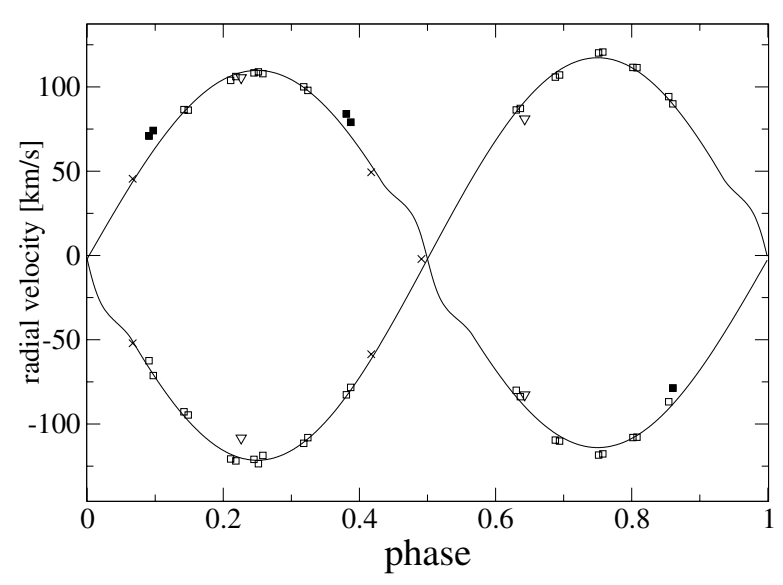

Fig. 4. Radial velocity curve of IO Aqr. Open squares represent cross correlation measurements in the $\mathrm{NaD}$ region and filled squares the rejected $\mathrm{NaD}$ observations; triangles present the $\mathrm{H}_{\alpha}$ measurement (obtained only from a single $\mathrm{H}_{\alpha}$ line). The velocities obtained near $5175 \AA$ come from observations by Ligeza at DDO observatory (Canada) and are marked with $x$ symbols. The solid lines represent the synthetic curves computed with the W-D code. The curve for component 1 has a maximum at phase 0.75 .

Table 5. Two solutions, based on our $\mathrm{NaD}$ radial velocity observations and ASAS I band photometry.

\begin{tabular}{ccc}
\hline \hline Parameter & Solution A & Solution B \\
\hline$i$ & $81.36 \pm 0.70$ & $81.63 \pm 0.62$ \\
$q$ & $1.068 \pm 0.006$ & $1.067 \pm 0.006$ \\
$A\left(R_{\odot}\right)$ & $10.96 \pm 0.04$ & $10.98 \pm 0.04$ \\
$\Omega_{1}$ & $5.90 \pm 0.22$ & $6.35 \pm 0.29$ \\
$\Omega_{2}$ & $5.91 \pm 0.20$ & $5.61 \pm 0.17$ \\
$l_{1}$ & $0.498 \pm 0.013$ & $0.420 \pm 0.013$ \\
$l_{2}$ & $0.502 \pm 0.013$ & $0.580 \pm 0.013$ \\
$T_{1}$ & \multicolumn{2}{c}{$6600 \mathrm{~K}$} \\
$T_{2}$ & $6400 \pm 300 \mathrm{~K}$ & $6425 \pm 300 \mathrm{~K}$ \\
$x_{12}$ & \multicolumn{2}{c}{0.4} \\
$g_{12}$ & \multicolumn{2}{c}{0.32} \\
$a_{12}$ & \multicolumn{2}{c}{0.5} \\
$\chi^{2} / d f$ & 1.26 \\
\hline
\end{tabular}

Table 6. Stellar parameters of the IO Aqr components (in solar units) for both A and B solutions, calculated from Wilson-Devinney model (Table 5).

\begin{tabular}{ccccc}
\hline \hline & ${\text { star } 1^{\mathrm{A}}}$ & star 1 $^{\mathrm{B}}$ & star 2 $^{\mathrm{A}}$ & star 2 $^{\mathrm{B}}$ \\
\hline Mass & $1.53 \pm 0.02$ & $1.53 \pm 0.02$ & $1.63 \pm 0.02$ & $1.64 \pm 0.02$ \\
$R_{\text {point }}$ & $2.32 \pm 0.15$ & $2.11 \pm 0.19$ & $2.42 \pm 0.11$ & $2.60 \pm 0.09$ \\
$R_{\text {side }}$ & $2.28 \pm 0.10$ & $2.09 \pm 0.13$ & $2.38 \pm 0.08$ & $2.54 \pm 0.06$ \\
$R_{\text {back }}$ & $2.31 \pm 0.10$ & $2.11 \pm 0.13$ & $2.41 \pm 0.08$ & $2.58 \pm 0.06$ \\
$R_{\text {pole }}$ & $2.26 \pm 0.10$ & $2.07 \pm 0.13$ & $2.36 \pm 0.08$ & $2.51 \pm 0.06$ \\
\hline
\end{tabular}

The masses and radii derived from the results of the final fit are listed in Table 6. Their covariances $A C_{x} A^{T}$ were computed from the covariance matrix $C_{x}$ of the parameters listed in Table 5, taking into account the linearized transformation matrix $A$. 


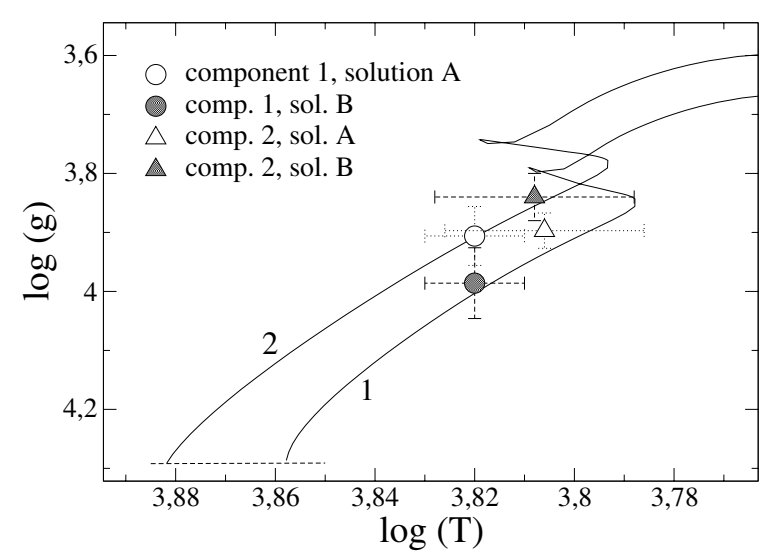

Fig. 5. Evolutionary tracks of both components. Circles present the first component while triangles denote the second one. As we can see solution B marked by filled symbols fits both curves better than solution A (open symbols). The temperature error bars for the first and the second components correspond to the error given by the Tycho 2 catalogue and to the error of our fit respectively.

\section{Age and distance}

\subsection{Distance and surface brightness}

For a simple estimation of the photometric parallax of IO Aqr we assume that the stars are spherical with radius $R_{\text {side }}$ and that their bolometric surface brightness is $\sigma T_{\mathrm{e}}^{4}$. The bolometric magnitude follows from the Stephan-Boltzman law (Lang 1978):

$M_{\mathrm{bol}}=42.37-5 \log \left(\frac{R}{R_{\odot}}\right)-10 \log T_{\mathrm{e}}$.

We adopt a $V$ magnitude of 8.87 and bolometric correction $B C_{V}=-0.14$ (Binney \& Merrifield 1998) corresponding to a temperature of $6500 \mathrm{~K}$. From the distance modulus $m-M$, neglecting the interstellar extinction, we obtain a distance of $256 \mathrm{pc}$ and a parallax $\pi=3.9$ mas. The distance obtained differs from the result of Hipparcos by $38 \%$. Taking the Hipparcos parallax of IO Aqr and our data in Tables 4 and 5 (radii $R_{12}$, luminosities $l_{12}$ and $I_{0}$-magnitude of the system) at their face value, from the formulae given by Bailey 1981 for the surface brightness of the components we obtain $S_{\mathrm{I} 1}=4.48 \pm 0.67$ and $S_{\mathrm{I} 2}=4.56 \pm 0.64$ for solution A and $S_{\mathrm{I} 1}=4.46 \pm 0.71$ and $S_{\mathrm{I} 2}=4.55 \pm 0.67$ for B.

\subsection{Evolutionary status}

To perform an independent check of the off-main-sequence status of the components of IO Aqr we performed a W-D fit forcing the stellar radii (and $\Omega_{1,2}$ ) to remain as small as on the main sequence for the derived masses, while relaxing the remaining parameters. Such a fit must be rejected as it led to a rise of $\chi^{2}$ by two orders of magnitude.

On the empirical mass-radius relation, the components of IO Aqr lie just off the main sequence (Gimenez \& Zamorano 1985), so both components ought be slightly evolved. In order to inspect this issue in more detail we plotted our parameters of the components of IO Aqr on the $\log \left(T_{\text {eff }}\right)-\log (g)$ diagram together with the evolutionary tracks of Kim et al. (2002) for $Z=0.02$ and respective masses (Fig. 5). The evolutionary tracks clearly favor solution $\mathrm{B}$. The primary component in solution A has too low $\log (g)$ while the secondary has stronger gravity than predicted by the theory. Taking our parameters at their face value we estimate the most likely age of the system to about $1.9 \mathrm{Gyr}$.

As pointed out by the referee one could resort to using spectra to assess our solutions A and B. They differ in the relative luminosity of the components. For as small a difference of the temperatures as suggested by our solutions, the main factor influencing the relative strength of lines is the luminosity of the components. Our spectra reveal considerable scatter of line ratios, with the average closer to 0.72 than to 0.99 , indicating a preference for model B. The difference between the two components is more noticeable in the $\mathrm{H}_{\alpha}$ spectra (Fig. 2).

\section{Conclusions}

New photometric and spectroscopic observations of the detached eclipsing binary IO Aqr, combined with ASAS photometry, have allowed us to to determine the orbital and physical parameters of the star. The two components of IO Aqr turned out to be close to their turn-off point from the main sequence. These properties open a potential application of IO Aqr for differential tests of the evolutionary tracks, at the particular phase near to core collapse, where models are particularly sensitive to their parameters. In order to tighten the uncertainty we need in the first place a more accurate parallax or, more likely, a more accurate temperature based on multicolor photometry, hence we postpone further discussion of this issue, for the time being.

Acknowledgements. W.D. and A.S.C. were supported by the KBN Grant 5P03D 002 20. We would like to thank Drs. Tomasz Kwiatkowski, Tomasz Plewa and Toma Tomov for their help with the reductions and modeling software. W.D. and A.S.C. would like to thank prof. K. Panov for the warm hospitality at NAO. We are most grateful to Piotr Ligeza on the one side, and to Regina Rudawska and Roman Hirsch on the other side, for their contribution of some spectroscopic and photometric observations, respectively.

\section{References}

Bailey, J. 1981, MNRAS, 197, 31

Binney, J., \& Merrifield, M. 1998, in Galactic astronomy, ed. J. Ostriker, \& D. Spergel (Princeton University Press)

Cernis, K. 1986, Vilnius Astron. Obs. Biul., 75, 31

Gimenez, A., \& Zamorano, J. 1985, Ap\&SS, 114, 259G

Kim, Y., Demarque, P., Yi, S., \& Alexander, D. 2002, ApJS, 143, 499

Kruszewski, A., \& Semeniuk, I. 1999, AcA, 49, 561.

Lang, K. R. 1978, Astrophysical Formulae (Springer-Verlag)

Perryman, M.A.C., Lindegren, L., Kovalevsky, J., et al. 1997, A\&A, 323,49

Plewa, T. 1992, Acta Astron., 38, 415

Plewa, T. 1988, Acta Astron., 42, 103

Pojmański, G. 1997, Acta Astron., 47, 467 http: //archive.princeton.edu/ asas/

Wade, R. A., \& Rucinski, S. M. 1985, A\&AS, 60, 471

Wilson, R. E., \& Devinney, E. J. 1971, ApJ, 166, 605 